Archives de sciences sociales des religions

$168 \mid 2014$

Bulletin Bibliographique

\title{
Une histoire séculière de la vie de Jésus
}

\section{François-André Isambert}

\section{(2) OpenEdition}

Journals

Édition électronique

URL : http://journals.openedition.org/assr/26304

DOI : $10.4000 /$ assr. 26304

ISSN : $1777-5825$

Éditeur

Éditions de l'EHESS

Édition imprimée

Date de publication : 31 décembre 2014

Pagination : 73-85

ISBN : 978-2-7132-2467-6

ISSN : 0335-5985

Référence électronique

François-André Isambert, "Une histoire séculière de la vie de Jésus », Archives de sciences sociales des religions [En ligne], 168 | 2014, mis en ligne le 30 mars 2018, consulté le 10 décembre 2020. URL :

http://journals.openedition.org/assr/26304; DOI : https://doi.org/10.4000/assr.26304

Ce document a été généré automatiquement le 10 décembre 2020.

(C) Archives de sciences sociales des religions 


\title{
Une histoire séculière de la vie de Jésus
}

\author{
François-André Isambert
}

\section{NOTE DE L'ÉDITEUR}

François-André Isambert, un des membres fondateurs du Groupe de sociologie des religions et de notre revue dans les années 1950, nous a fait parvenir cette information sur un essai inédit émanant de son ancêtre homonyme, François-André Isambert (1792-1857). Il s'agit d'une étude historique sur Jésus à l'instar du Jesusleben de David Friedrich Strauss (1835) et de la célèbre Vie de Jésus d'Ernest Renan (1863). Ce volumineux document a été récemment déposé par la famille au Département des Manuscrits de la Bibliothèque Nationale de France. Souhaitons que cette œuvre, jusqu'alors non livrée au domaine public, suscite l'intérêt de l'historien. 


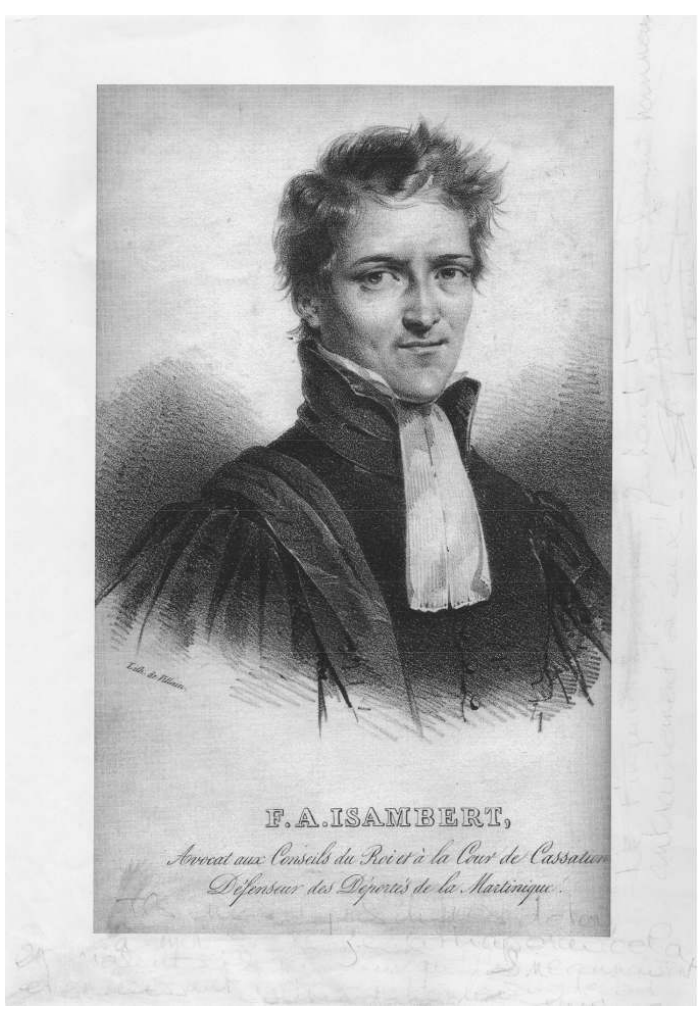

«Recherches d'un adepte du Christian isme sur la vie de Jésus et de ses disciples et sur l'histoire religieuse des premier et second siècles de notre ère, par I. de S. » (daté de 1843). Ainsi s'intitule une liasse de plus de 500 pages, au milieu d'un dossier au nom de François-André Isambert (1792-1857) déposé récemment au Département des Manuscrits de la Bibliothèque Nationale de France ${ }^{1}$. On a donc affaire, comme l'atteste le testament olographe de $1848^{2}$, à un inédit du juriste, homme politique et historien bien connu comme auteur du Recueil des Anciennes Lois Françaises en 30 volumes et de nombreux travaux de Droit, d'Histoire et de Géographie. Le titre (sans doute rédigé en fin de rédaction) a été composé soigneusement: le terme « Recherches » indique bien qu'il ne s'agit pas d'une «Vie de Jésus» au sens habituel, histoire-récit reconstituant l'enchaînement des faits de la vie de Jésus-Christ, mais d'une réflexion sur les traces aussi nombreuses que possible, profanes aussi bien que sacrées, laissées par les fondateurs, sur leur validité et sur les conclusions qu'on pouvait alors tirer des discussions qu'elles suscitaient.

2 On trouve d'assez nombreux travaux de ce genre, à la fin du XVIII et au XIX siècle en Angleterre avec, par exemple The Credibility of the Gospel History (1730) de Nathaniel Lardner et en Allemagne avec notamment la Jesusleben (1835) de David Friedrich Strauss, traduite en français en 1839 par Littré. C'est dans cette ligne que l'on peut situer les Recherches. Ces ouvrages, sortes de Bibles modernes, ne se disaient pas anti-chrétiens, mais se voulaient refonder un christianisme démystifié. Ils n'en rencontraient pas moins une hostilité, voire la condamnation de la part des Églises. En France, au milieu du XIX ${ }^{\mathrm{e}}$ siècle, à part des livres généraux et généralement partisans comme L'origine de tous les cultes ou la religion universelle (1795) de Charles-François Dupuis, seules paraissaient des histoires religieuses fondées sur des textes canoniques, comme celle de l'Abbé Glaire.

D'après ses propres notes de 1843, l'auteur des Recherches aurait commencé le rassemblement de son information, l'interprétation de celle-ci et sa critique pour lui seul, 
puis aurait songé à publier sous un pseudonyme, mais aurait jugé inopportun de le faire à ce moment, craignant les polémiques voire les sanctions. Il a alors interrompu son travail sans le terminer, laissant en blanc, ou presque, de nombreux chapitres, par exemple ceux qui concernaient le devenir des apôtres après la mort de Jésus. Le testament olographe permet de préciser cette réserve: son rédacteur écarte les funérailles religieuses, catholiques ou protestantes, non seulement par humilité mais parce qu'il réprouve tout esprit de secte lui opposant une conception unitaire du christianisme (à ne pas confondre avec les adeptes du groupement des « unitariens » hostiles à la Trinité). Il charge ainsi un « pasteur libéral » de le porter en terre.

Né dans une famille d'agriculteurs beaucerons aisés et catholiques convaincus, il fit des études qui le menèrent du Collège de Chartres au Lycée Louis-le-Grand et à l'École Normale Supérieure, à laquelle il préféra la Faculté de Droit, où il fut membre d'une Loge d'orientation républicaine et fréquenta les Carbonari. Il fut sous la Restauration un des principaux avocats de l'Opposition, avec la défense du Général Berton, d'Armand Carrel et des Quatre Sergents de la Rochelle. Il fut ensuite le défenseur ardent des «Hommes de Couleur ", noirs et mulâtres martiniquais condamnés aux galères pour leur revendication d'indépendance, ce qui lui valut une grande popularité Outre-Mer. Dans le même esprit, il prit, sous la Monarchie de Juillet, la tête du mouvement pour l'Abolition de l'Esclavage qui aboutit à la loi de 1848 actualisant la législation française dans ce sens. Au même moment, il tenait le secrétariat de la commission de la Chambre pour le Suffrage Universel.

Cet homme d'action démocrate était un érudit. Étudiant au Collège de France, il avait été élève de Pierre Daunou, promoteur de l'histoire critique. Au Collège de France, aussi, il avait travaillé avec Jean-Baptiste Gail à son Histoire de l'Orient ancien et l'avait enrichi de cartes géographico-historiques. Membre actif de la Société de Géographie, il poursuivit ses travaux de géographie et d'histoire de l'Est-méditerranéen, notamment dans le Bulletin de la Société. Il lut et même traduisit nombre d'auteurs grecs et latins et quantité d'auteurs juifs. Il traduisit les Analecta de Procope et écrivit lui-même une Histoire de Justinien, empereur $\mathrm{du} \mathrm{VI}^{\mathrm{e}}$ siècle et dont le règne fut particulièrement riche aux points de vue juridique et ecclésiastique. Il traduisit pour lui-même une grande partie de l'œuvre de Flavius Josèphe et L'Histoire ecclésiastique d'Eusèbe de Césarée. Mais l'état d'es-prit de ses contemporains le préoccupait au moins autant. Dans L'État religieux de la France et de l'Europe (1843), écrit avec Charles-Philibert de Lasteyrie et Arthur Condorcet O'Connor, il tente la statistique contemporaine des membres des confessions des différents pays d'Europe, avec, lorsque c'est possible, une différenciation des types d'adhésion (avantgoût de la sociologie religieuse moderne). Lui et ses co-auteurs voient aussi émerger ce qu'ils mettent sous le nom de "Déisme », sorte de religion commune, qu'il appartiendrait à l'État, et plus particulièrement à l'Université d'en assurer le culte public et l'enseignement et d'en protéger l'expression de toute confession particulière. Quoique non proclamé, ce « déisme » serait déjà devenu la base de toute pensée objective en matière religieuse.

6 Les Recherches commencent par une confidence: à ses débuts, l'auteur ne mit pas en doute la justesse des enseignements qu'il reçut. Mais ses études ultérieures et les lectures qu'il fit à cette occasion le firent douter de tout ce qu'il avait appris. Il éprouva alors le besoin de se « raffermir » contre le «scepticisme du siècle » et pour cela de se rattacher plus fermement à cette "Réforme » religieuse qu'a été le christianisme primitif. Récrire un nouveau Credo, inspiré par l'ancien, mais rectifié en fonction des conquêtes de la raison. L'opposition morale entre christianisme et paganisme étant une idée admise, elle 
est développée ici pour l'exemple par une citation de plusieurs dizaines de pages de Thomas Jefferson. Nous sommes directement ramenés aux textes du Nouveau Testament et aux Pères de l'Église. Ces écrits doivent être relus, mais cette fois au «crible de la critique ordinaire » et en les confrontant aux textes profanes. Prenant la forme de récits et se donnant pour historiques, ils seront soumis à des jugements de réalité. On peut ainsi parler d'une histoire séculière des textes sacrés où la critique qui y règne n'est autre que l'examen que doit effectuer l'historien en toutes circonstances.

Dans son titre-même, notre auteur s'était déclaré « adepte du christianisme ». C'est en tant que tel qu'il prenait la plume. Allait-il tenter dès lors d'ajuster ses convictions les plus intimes à ce qu'il croyait pouvoir établir comme vrai dans une pensée séculière ? Mais pour lui, la question ne semble pas se poser tant il identifie le Christianisme à la Vérité. C'est évidemment sur le plan éthique qu'une telle identité peut être concevable. C'est ce sur quoi un certain nombre de "grands hommes" de l'époque sont tombés d'accord et particulièrement Jefferson longuement cité. Afin de n'exclure personne, Isambert nomme « chrétien » aussi bien celui qui croit que Jésus fut « inspiré par Dieu » que celui qui croit « qu'il est Fils de Dieu », donc Dieu lui-même. On sait que, pour l'Église, c'est la seconde option qui correspond à la Vérité et elle en fait un dogme. Les Églises protestantes la font en général figurer dans leur Profession de Foi. Cela, l'historien peut l'enregistrer, alors que, dans sa démarche scientifique il est obligé de s'abstenir sur la véracité de l'objet lui-même. C'est par la voix évangélique de Jean, selon laquelle «le Verbe s'est fait chair", que la filiation divine est proclamée. Mais la croyance et l'expression de la croyance sont du domaine séculier et l'auteur a de fortes raisons pour penser que ni les disciples, ni les premiers chrétiens ne croyaient en la divinité de Jésus de Nazareth. Un écho d'une Église grecque, au milieu du II ${ }^{\mathrm{e}}$ siècle rapporte qu'on comptait alors deux sortes de chrétiens, ceux qui croyaient en la filiation divine et ceux qui n'y croyaient pas, mais voyaient néanmoins en Jésus le Messie. La question de la généalogie interfère avec la précédente : si on suit l'Évangile de Matthieu, on remonterait de Jésus à Joseph, son père, et au-delà jusqu'à Abraham, alors que les autres Évangiles le font seulement descendre de Marie. Ainsi, pour un point central du christianisme, l'auteur cherche à éclairer les raisons qui ont poussé les uns et les autres à prendre position tout en se donnant à lui-même une liberté raisonnée de conviction.

Suivant la recommandation de Daunou, l'auteur fait de la critique des sources l'essentiel de la critique historique. Il y a lieu, selon lui, de distinguer, parmi celles-ci, les « traditions », les « monuments » et les « relations originales » ou témoignages directs. La distinction entre passages canoniques et apocryphes, elle, n'est pas utilisable par l'historien, quant à la véracité du contenu, les critères sur lesquels elle repose étant purement ecclésiastiques. Aussi l'historien ne se privera-t-il pas d'avoir recours aux apocryphes, en particulier là où les canoniques restent muets. Tel est le cas en particulier de l'Enfance de Jésus qui a donné naissance dès les premiers siècles à une multitude de récits légendaires. Parmi ceux-ci, Isambert épingle ceux du Sefer Toledot Yeshu ( « Livre des engendrements de Jésus »), pamphlet juif antichrétien du Moyen Âge caricaturant l'origine de Jésus. Il entretient à ce sujet une correspondance avec Salomon Munk, un érudit juif qui montre le peu de sérieux que l'on peut accorder à une telle source. Tous les apocryphes toutefois ne sont pas de la même trempe et l'auteur met en évidence une tradition juive et une tradition islamique qui méritent considération. Il faut bien passer par la notion de «tradition » pour parler des Évangiles. L'auteur a peu de mal à confirmer l'idée qui se répandait en son temps et selon laquelle les livres canoniques ne résultaient 
pas des "relations originales", mais, vu les dates attribuables à leur rédaction, appartenaient au genre des traditions. C'est-à-dire que chaque Évangile, à moins d'être la copie partielle d'un autre, serait la transcription d'un texte oral se transmettant par la mémoire. On pourrait placer Marc comme le premier d'une suite comprenant Luc et Matthieu, inspirés des précédents. Jean, tout en s'accordant sur bien des points avec les autres, apporte une note différente où on reconnait des accents néo-platoniciens.

Finalement, avec prudence, Isambert propose un schéma qui lui paraît le plus vraisemblable: cela commencerait avec les Esséniens, parmi lesquels Jean-Baptiste, réunissant autour de lui des assemblées pour des prédications suivies de baptêmes. Jésus se serait fait baptiser, après quoi, inspiré par Dieu, il aurait commencé sa propre campagne de prédications. Celles-ci auraient duré plusieurs années et Jésus aurait atteint la cinquantaine. Les circonstances de sa mort sont l'objet d'affirmations contradictoires. Plusieurs d'entre elles ne parlent guère de la Passion, sur laquelle, pourtant, les Évangiles canoniques s'accordent, ainsi que l'Histoire ecclésiastique d'Eusèbe.

Aujourd'hui, un ouvrage comme celui-ci peut sembler démodé : d'une part d'importantes découvertes documentaires ont renouvelé des questions parmi les plus importantes (comme celle des Manuscrits de Qumram); d'autre part, l'atmosphère a beaucoup changé entre les «biblistes", songeant maintenant plus à la complémentarité de leurs travaux qu'à leur adversité. Il ne semble pas inintéressant néanmoins de voir où pouvaient en arriver des savants, dans un questionnement très complexe, au moment même où, en France, l'Histoire prenait un nouvel essor décisif. Plus concrètement, le chercheur d'aujourd'hui trouvera dans ces Recherches : un inventaire fort détaillé des sources écrites latines et grecques sur la vie de Jésus-Christ (avec des excursus dans d'autres langues) ; un plan des diverses phases de la vie de Jésus situées dans le cadre social où il a vécu; une évocation des principaux problèmes que sa vie a suscités et des réponses qui furent données. L'ouvrage, a-t-on bien dit, est inachevé et fort incomplet. Aussi le traitera-t-on essentiellement comme une suite de questions, mais aussi de réponses, souvent contradictoires, entre lesquelles il propose un choix raisonné et non dogmatique.

\section{Document 1}

\section{TABLE DES MATIÈRES} et de ses discipleset sur l'histoire religieuse des premier et second siècles de notre ère, par I. de S. "

NB : Table établie par Viviane Isambert-Jamati à partir des feuillets du manuscrit original. La pagination indiquée est celle de la transcription tapuscrite. L'inégalité de volume entre chapitres reflète l'état d'inachèvement du manuscrit. 


\section{Introduction}

16 p. 2

\section{Livre I. Opinion critique des Grands Hommes sur les fondements du Christianisme}

17

\section{Livre II. De l'autorité historique des évangiles}

\section{Livre III. De l'histoire religieuse des Juifs depuis les} temps anciens jusqu'à la naissance de Jésus-Christ en l'an 7 de notre ère 
Chapitre 3. Jésus contemporain d'Alexandre Jannée, roi Asmonéen des Juifs vers l'an 90 avant notre ère (traditions talmudiques)

Chapitre 4. Tradition purement juive : Toldos Jeschu

Chapitre 5. Extraits du Toledoth-Jeschu. Jésus contemporain d'Alexandre Jannée; quatrième tradition extraite du Maasé Talouy

Chapitre 6. Histoire religieuse des Juifs depuis la mort de la veuve de Jannée (an 61) jusqu'à l'an 37 de notre ère

Chapitre 7. Réponse à l'objection tirée d'Origène sur le passage relatif à Jacques, frère de Jésus

Chapitre 8. Histoire religieuse des Juifs sous Hérode $1^{\mathrm{er}}$, de l'an 37 avant notre ère jusqu'au commencement des traditions évangéliques, an 17 avant notre ère

Chapitre 9. Histoire religieuse des Juifs depuis l'an 17 avant notre ère jusqu'à la fin du règne $\mathrm{d}^{\prime}$ Hérode $1^{\mathrm{er}}$

Chapitre 10. Opinion de MM. Salvador et Franck, écrivains israélites contemporains sur les opinions religieuses des Juifs vers l'époque de l'avènement de Jésus-Christ

Chapitre 11. Erreurs chronologiques d'Eusèbe et de Denys-le-Pieux, inventeur de l'ère de la nativité de Jésus-Christ

Chapitre 12. Ce qu'enseignent les monuments; fausses interprétations des prophéties

Chapitre 13. Sur la naissance de Jésus-Christ, compléments

\section{Livre IV. Histoire religieuse des Juifs de la naissance de Jésus-Christ, l'an VII de notre ère à son baptême en I'an XXIX}

p. 283

Chapitre 1. Histoire religieuse des Juifs depuis la mort d'Hérode $1^{\mathrm{er}}$, an 4 avant notre ère, jusqu'à la mort de l'Empereur Auguste, an 12 de notre ère

Chapitre 2. De la deuxième tradition évangélique sur la naissance de Jésus-Christ comparée avec l'histoire: Jésus né l'an du recensement de Cyrénius, l'an 4 de notre ère, après la déchéance $d$ 'Archelaüs

Chapitre 3. Témoignage de l'Empereur Julien

Chapitre 4. Explication de la contradiction des généalogies par Africanus, cité par Eusèbe

Chapitre 5. Différence de 14 ans entre l'évangile selon Matthieu et l'évangile selon Luc relativement à la naissance de Jésus-Christ

52 Chapitre 6. Opinion des Pères de l'Église antérieurs à Eusèbe sur l'époque de la naissance de Jésus-Christ

Chapitre 7. Des passages d'Eusèbe relatifs à la même période, c'est-à-dire de la naissance de Jésus-Christ à son baptême, an 19 de Tibère.

Chapitre 8. Opinion d'Epiphane sur la naissance de Jésus-Christ

Chapitre 9. Époque de la naissance selon les Apocryphes

Chapitre 10. Opinion d'Irénée sur l'époque de la naissance de Jésus-Christ 

naissance de Jésus-Christ Jésus-Christ. De l'évangile des Marcionites

\section{p. 349} de notre ère l'an 29 à l'an 36 de notre ère tétrarque de la Galilée et de la Pérée en l'an 36

p. 380

Plan esquissé par l'auteur

Chapitre 4. Suite de la vie publique de Jésus ${ }^{3}$

p. 501

Chapitre 11. De l'esprit de superstition qui régnait dans l'Empire Romain à l'époque de la

Chapitre 12. Époque réelle de la naissance de Jésus-Christ

Chapitre 13. Histoire religieuse des Juifs depuis la mort d'Auguste jusqu'à la quinzième année de Tibère (an 28 de notre ère : ans 7-21 de Jésus-Christ)

Chapitre 14. Des récits évangéliques relatifs à la période écoulée entre l'an 16 et l'an 24 de notre ère, sous Tibère. Du baptême de Jésus-Christ

Chapitre 15. Pourquoi Nicolas de Damas n'a pas parlé de la naissance et de la jeunesse de

Chapitre 16. Du passage de Josèphe relatif à Jésus-Christ

\section{Livre V. De l'interprétation du baptême, ou du prophète Jean, l'an 25 à l'an 35}

Chapitre 1. Durée de la mission de Jean-Baptiste de l'an 28 à l'an 35 de notre ère

Chapitre 2. Récits évangéliques relatifs à la mission et à la mort de Jean-Baptiste, 29-35

Chapitre 3. De l'institution du baptême et de la prédication de Jean-Baptiste

Chapitre 4. Histoire de la mort de Jean-Baptiste, ou histoire religieuse de la Judée et de

Chapitre 5. De la captation du Prophète Jean-Baptiste par ordre d'Hérode (Antipas)

\section{Livre VI. De la vie publique de Jésus, de l'an 30 de son âge (an 37-38 de l'ère chrétienne) à la mort de Jésus (an 60 ou 61)}

Chapitre 1. Époque préparatoire, de l'an 6 à l'an 35 de notre ère

Chapitre 2. De la durée de la vie publique de Jésus

Chapitre 3. Impossibilité de concilier la mort de Jésus-Christ à 50 ans de son âge avec la tradition qui la place sous Ponce-Pilate et Tibère, de l'an 33 à l'an 36 ou 37 de notre ère

Chapitre 5. Du choix des Apôtres et de leur mission pendant la vie de Jésus

\section{Livre VII. De la mise à mort de Jésus par les Juifs sous le Pontife Anne ou Ananus an 60-61 de notre ère}




\section{Livre VIII. De la prédication apostolique}

p. 523

Plan et rappel du texte précédent

Chapitre 1. De la prééminence de Pierre

Chapitre 2. De la dispersion et de la mort des apôtres

Chapitre 3. Des personnages apostoliques

Chapitre 4. De l'époque où la doctrine de Jésus fut répandue dans l'Empire

Chapitre 5. Des hérétiques, ou dissidents

Chapitre 6. Progrès du Christianisme. De l'époque où l'on a cessé d'appeler Jésus « fils de l'homme »

\section{Livre IX. De l'institution définitive des Évêques et des Conciles} réformateur du Judaïsme

Chapitre 2. Interpolation relative à la Trinité

(Pas de Chapitre 3 à 8 )
Livre X. Histoire de l'Église pendant le Troisième Siècle. Charte de la liberté des cultes proclamée par Constantin, A. 312

p. 539

Chapitre 1. Chrétiens de deux classes, au milieu du Second Siècle : ceux qui croient à la divinité de Jésus-Christ, et ceux qui, sans y croire, le regardent comme le Messie,

Chapitre 9. Des Pères apologétiques et du Canon des Évangiles 


\section{Notes préparatoires à l'ouvrage}

p. 543

\section{Correspondance avec S. Munk}

p. 565

\section{Index des noms (Antiquité)}

p. 569

\section{Index des noms (Temps modernes)}

105 p. 575

\section{Index des lieux}

p. 583

\section{Document 2}

(En marge) :

Point de cérémonie catholique à mon décès.

Point de députation de la chambre de Cassation

Signé: Isambert

\section{Signé et daté du $1^{\text {er }}$ décembre 1848} à ma naissance, dans le village d'Aunay (Eure-et-Loire) le 30 novembre 1792, quand les cultes étaient libres. Je me suis occupé toute ma vie durant de régler mes croyances. J'ai fait ma première communion dans la paroisse de Saint-Pierre de Chartres, avant l'âge de discernement. Il est résulté de l'examen consciencieux que j'en ai fait: 
$\left.1151^{\circ}\right)$ Que le clergé enrôle les enfants dans la communion à dessein, avant l'âge de discernement fixé par nos lois à 15 ans accomplis (art.79, Code criminel), afin d'empêcher l'effet de leur raisonnement.

$\left.1162^{\circ}\right)$ Que cette religion n'est pas plus vraie que la Religion de Mahomet, que la Brahmanisme et le Bouddhisme et celle de [...], plus ancienne qu'elles et comprenant un aussi grand nombre de sectateurs, quoique sa morale soit plus dure et plus conforme aux règles de la Civilisation.

$\left.1173^{\circ}\right)$ Que les évangiles [...] ne sont point des écrits authentiques; qu'ils ont été remaniés ainsi que l'avoue Jérôme dans sa lettre au pape Daniel en tête de la traduction latine qu'on appelle la Vulgate. Que ces évangiles, arbitrairement limités au nombre de 4, ne datent que de l'an 170 ou 180 de l'ère vulgaire; qu'eux, et surtout l'écrit intitulé Les actes des Apôtres sont pleins de miracles supposés et d'erreurs de date. Que les généalogies de Jésus-Christ sont contradictoires et fausses, reconnues comme telles par Paul. Que Jésus de Nazareth, né peut-être au village de Bethléem, près de Jérusalem, l'an du recensement, ou 8 de notre ère, après la réunion de la Judée à l'Empire Romain, n'a pu établir une secte nouvelle, pour la réforme du Judaïsme, après Jean-Baptiste, que par des efforts prolongés, pendant une vie d'environ 50 ans. Qu'il est mort sous Ananus II, et par ordre de ce Sanhédrin, vers l'an 60-62 de notre ère moderne. Que la religion nouvelle, dont parle l'historien Josèphe, n'avait encore aucune consistance suffisante pour avoir le nom de Christianisme, lorsque l'ardeur des opinions politico-religieuses, exaltée par l'oppression des Préfets Romains, excita la Guerre des Juifs contre les Romains; qu'aussi dans cette guerre, on ne trouva jamais aucune Église établie.

118 La religion nouvelle fut une réaction légitime des opprimés contre l'oppression et la barbarie des Romains, après la prise de Jérusalem, et contre le polythéisme corrupteurs des mœurs.

119 Paul de Tarse paraît avoir autant influé que Jésus sur la formation de cette religion en appelant les Gentils, ou les Grecs à coopérer à la Réforme. Paul, auteur de la doctrine abstraite et mystique de la Trinité, paraît avoir vécu dans le Second Siècle et avoir été connu de Lucien de Samosate qui a parlé des Chrétiens comme des adversaires de l'athéisme. Le premier monument authentique de l'existence des Chrétiens est la lettre de Pline, Gouverneur de la Bythinie, à l'empereur Trajan.

120 La persécution contre les Chrétiens ne paraît avoir commencé que du temps de l'Empereur Commode, après que le philosophe Celse l'ait crue assez importante pour mériter d'être combattue. Jusque-là, il n'y avait que des controverses plus ou moins vives contre les (...) formulées contre les Juifs convertis. La persécution de Néron ne paraît avoir porté que sur des Juifs disputant sur le Christ, comme ils avaient déjà fait du temps de Claude, et parce qu'ils étaient odieux au genre humain par leur intolérance.

121 Le Christianisme a été un grand progrès par rapport aux absurdités et aux corruptions du Paganisme; mais de persécutés ils devinrent persécuteurs, grâce à la protection de Théodose, an 364 et années suivantes. C'est ce cruel Empereur qui a commencé la série de lois de persécution, qui ont fait couler des flots de sang au nom du Catholicisme; c'est lui qui a changé le doux nom de Chrétiens, qui n'emportait pas avec lui l'exclusion de la liberté de conscience et de culte, proclamée en 312 par Constantin, au profit de toutes les croyances de l'Empire.

122 La tolérance n'a commencé qu'en France en 1789, car les sectateurs de Luther et de Calvin se sont aussi montrés persécuteurs, là où ils se sont trouvés les plus forts.

123 C'est pourquoi, en tant que Chrétien unitaire, je veux que mon corps ne soit porté à aucune église, catholique, luthérienne ou calviniste, n'étant en communion qu'avec les croyants qui respectent chez autrui la liberté de conscience. Mais ayant en horreur l'athéisme et ses sectateurs, tels que Proudhon et autres démagogues, ou les Egoïstes qui ne croient à rien. 
124 Je veux, comme Daunou et comme plusieurs de mes collègues à la Cour de Cassation, qu'on ne fasse aucune cérémonie religieuse à mon convoi, puisqu'il n'y a pas d'Église de mon culte; ni aucune députation officielle, me contentant du suffrage de l'amitié.

Je croyais que la République de 1848 nous aurait indemnisés du mal qu'elle nous a fait en maintenant l'égalité entre les cultes; mais à ma grande surprise j'ai vu Cavaignac et ses Ministres se prosterner devant l'Idole, et violer le texte et l'esprit de la Constitution du 4 novembre 1848, dans l'intérêt de leurs ambitions. "O fluttas hominum mentes, o pectora caeca "5. Je regrette d'être obligé, au milieu du XIX $x^{e}$ siècle, d'expliquer ainsi mes sentiments.

Je veux que ma chère Eugénie, qui m'a soutenu par sa tendresse pendant les longues tribulations de ma vie depuis notre mariage, pendant ma carrière d'avocat et dans ma carrière politique, ait après ma mort un revenu net et vierge de quatre mille francs, et que tout complément lui soit fourni sur ma succession, si sa requête pour la succession de Madame Vincent, sa cousine, ses droits matrimoniaux et sa pension de veuve ne suffisaient pas pour atteindre ce chiffre.

Je veux même que ce chiffre soit porté à six mille francs si elle reçoit la pension de 3000 francs comme veuve de Conseiller. Car cette pension est un bien personnel résultant de la collaboration. Nous espérions que notre chère fille Caroline nous survivrait. Sa perte a versé sur notre vie une amertume que rien ne peut détruire. Je prie mes trois fils de tout faire pour assurer le bonheur de nos chères petites-filles Alice et Nelly et de venir à leur secours si mon gendre, leur père, avait le malheur de perdre la dot de leur mère. Anténor est dans une position honorable; il est aimé et considéré. Je le conjure de travailler au droit, et de se tenir en activité, de manière à parvenir aux magistratures supérieures. Son excellente femme le secondera toujours dans cette voie.

Alfred est appelé à prendre une place avantageuse au Barreau, si sa mauvaise santé l'en empêche. Il dépend de lui, par une vie sévère, d'améliorer sa santé, et peut-être d'arriver de bonne heure à la vie politique. Mais qu'il ne se presse pas; cette carrière est pleine d'angoisses et de périls. Mon fils Émile est plein de moyens et de santé; il a bien débuté dans les études médicales; il a montré de la maturité, et il a tenu une excellente conduite dans son essai de diplomatie à Florence, où je l'avais envoyé pour le former; mais je désire qu'il ne suive pas cette carrière et qu'il revienne à la médecine et aux sciences.

133 Je laisse de nombreux manuscrits; je désire vivement que sous mon nom, ou si les préjugés religieux sont trop forts, sous celui de M. J de Sumerville, on publie les travaux que j'ai faits sur la fondation du Christianisme. La vérité à ce sujet n'est pas connue et elle mérite de l'être. J'en ai indiqué les bases dans l'article Christianisme qui a paru sous mon nom dans l'Encyclopédie de Didot, et dans les deux volumes publiés en 1845 avec le Gén. O'Connor et Ch. De Lasteyrie dans le Journal de la Liberté Religieuse, dont Goubaud fut le gérant. Il faudra consulter M. Alfred Maury sur mes manuscrits religieux, et sur la publication de ma traduction de Flavius Josèphe.

Paris, ce $1^{\text {er }}$ décembre mil huit cent quarante-huit, l'an de la fuite du pape de Rome.

Isambert 


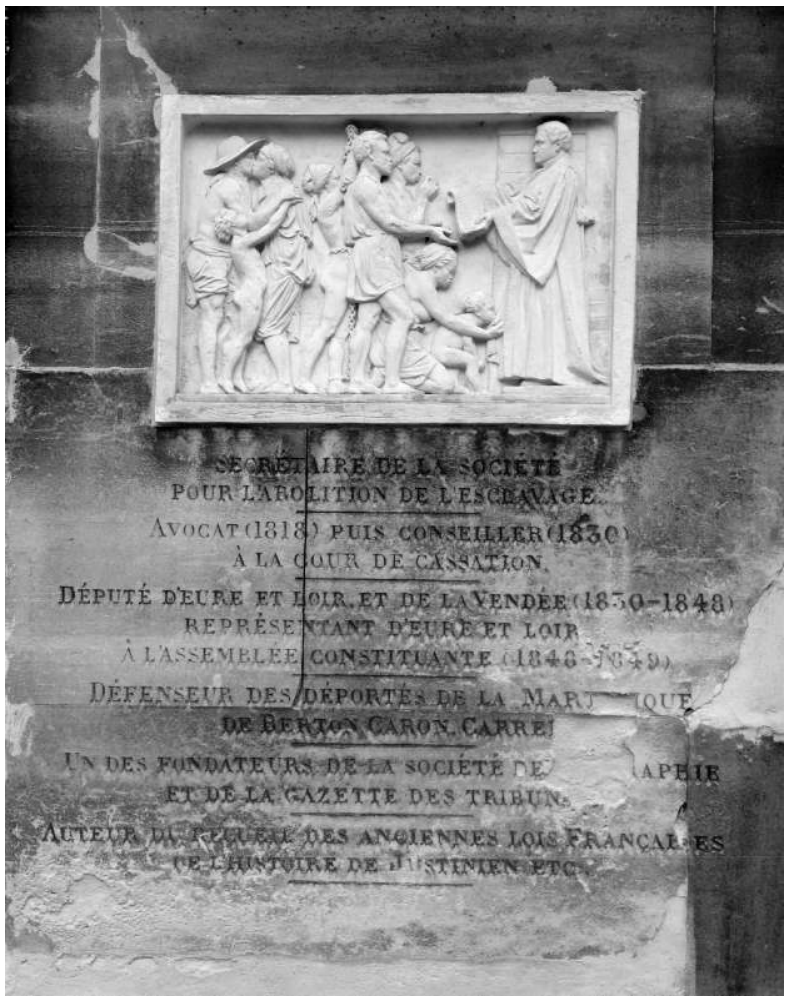

Pierre Lassave, CéSor

\section{NOTES}

1. Le dépôt (cote NAF 28624) rassemble plusieurs fascicules manuscrits à l'écriture pas toujours lisible. Pour rendre l'œuvre utilisable, Viviane Isambert-Jamati l'a classée et transcrite sur traitement de texte (pièce incluse dans le dépôt à la BNF). Le document 1 indique sa table des matières.

2. Transcrit dans le document 2.

3. Ce chapitre 4 du Livre VI comporte $17 \S$, chacun portant un titre et s'étendant sur plusieurs pages.

4. L'auteur a écrit " avant », mais c'est évidemment un lapsus.

5. Ô esprits fluctuants des hommes! Ô cœurs aveugles! 


\section{AUTEUR}

FRANÇOIS-ANDRÉ ISAMBERT

École des Hautes Études en Sciences Sociales 